\title{
Some Conclusions on Cayley Digraphs and Their Applications to Interconnection Networks ${ }^{\star}$
}

\author{
Wenjun Xiao ${ }^{1,2}$ and Behrooz Parhami ${ }^{3}$ \\ 1 Dept. of Computer Science, South China University of Technology, \\ Guangzhou, 510641,P.R. China \\ 2 Dept. of Math., Xiamen University, Xiamen, Fujian, 361005, P.R. China \\ wjxiao@scut.edu.cn \\ 3 Department of Electrical and Computer Engineering, University of California, \\ Santa Barbara, CA 93106-9560, USA. \\ parhami@ece.ucsb.edu
}

\begin{abstract}
In this short communication, we survey the relationships between Cayley digraphs and their subgraphs and coset graphs with respect to subgroups and obtain some general results on homomorphism and broadcasting between them. We also obtain a factorization of Cayley digraphs on subgraphs. We discuss the applications of these results to well-known interconnection networks. These conclusions possess potential application to grid computing.
\end{abstract}

\section{Introduction}

It is known that Cayley (di)graphs and coset graphs are excellent models for interconnection networks [1], [2], [5]. Many well-known interconnection networks are Cayley (di)graphs or coset graphs. For example, hypercube, butterfly, and cube-connected cycles networks are Cayley graphs, while de Bruijn and shuffleexchange networks are coset graphs [5]. As suggested by Heydemann [5], general theorems are lacking for Cayley digraphs and more group theory has to be exploited to find properties of Cayley digraphs. In this paper, we consider the relationships between Cayley (di)graphs and their subgraphs and coset graphs with respect to subgroups and obtain some general results on homomorphism between them. We provide several applications of these results to well-known interconnection networks. Before proceeding further, we introduce some definitions and notations related to (di)graphs, Cayley (di)graphs in particular, and interconnection networks. For more definitions and basic results on graphs and groups we refer the reader to [3], for instance, and on interconnection networks to [6], [7]. Unless noted otherwise, all graphs in this paper are directed graphs. A digraph $\Gamma=(V, E)$ is defined by a set $V$ of vertices and a set $E$ of arcs or directed edges. The set $E$ is a subset of elements $(u, v)$ of $V \times V$. If the subset $E$ is symmetric, that is, $(u, v) \in E$ implies $(v, u) \in E$, we identify two opposite

\footnotetext{
* This work was supported by the Natural Science Foundation of China and Fujian Province.
} 
$\operatorname{arcs}(u, v)$ and $(v, u)$ by the undirected edge $(u, v)$. We then obtain a graph. Let $G$ be a (possible infinite) group and $S$ a subset of $G$. The subset $S$ is said to be a generating set for $G$, and the elements of $S$ are called generators of $G$, if every element of $G$ can be expressed as a finite product of their powers. We also say that $G$ is generated by $S$. The Cayley digraph of the group $G$ and the subset $S$, denoted by $\operatorname{Cay}(G, S)$, has vertices that are elements of $G$ and arcs that are ordered pairs $(g, g s)$ for $g \in G, s \in S$. If $S$ is a generating set of $G$ then we will say that $\operatorname{Cay}(G, S)$ is the Cayley digraph of $G$ generated by $S$. If $1 \notin S$ (1 is the identity element of $G$ ) and $S=S^{-1}$, then $\operatorname{Cay}(G, S)$ is a simple graph. Assume that $\Gamma$ and $\Sigma$ are two digraphs. The mapping $\phi$ of $V(\Gamma)$ to $V(\Sigma)$ is a homomorphism from $\Gamma$ to $\Sigma$ if for any $(u, v) \in E(\Gamma)$ we have $(\phi(u), \phi(v)) \in E(\Sigma)$. In particular, if $\phi$ is a bijection such that both $\phi$ and the inverse of $\phi$ are homomorphisms then it is called an isomorphism of $\Gamma$ to $\Sigma$. Let $G$ be a (possible infinite) group and $S$ a subset of $G$. Assume that $K$ is a subgroup of $G$ (denoted as $K \leq G)$. Let $G / K$ denote the set of the right cosets of $K$ in $G$. The (right) coset graph of $G$ with respect to the subgroup $K$ and subset $S$, denoted by $\operatorname{Cos}(G, K, S)$, is the digraph with the vertex set $G / K$ such that there exists an $\operatorname{arc}\left(K g, K g^{\prime}\right)$ if and only if there exists $s \in S$ and $K g s=K g^{\prime}$. The following basic result is easily verified.

Theorem 1. The mapping $\phi: g \rightarrow K g$ is a homomorphism from $\operatorname{Cay}(G, S)$ to $\operatorname{Cos}(G, K, S)$ for $g \in G$.

\section{Main Results}

The tense product $\Gamma \times \Sigma$ denotes the digraph with the vertex set $V(\Gamma) \times V(\Sigma)$ and the arc set $\left\{\left(\left(x_{1}, x_{2}\right),\left(y_{1}, y_{2}\right)\right) \mid\left(x_{1}, y_{1}\right) \in E(\Gamma),\left(x_{2}, y_{2}\right) \in E(\Sigma)\right\}$.

Let us assume that the group $G$ satisfies $G=N K$, where $N$ is a normal subgroup of $G$ (denoted by $N \triangleleft G$ ), $K \leq G$, and $N \cap K=1$; that is, $G$ is the semidirect product of $N$ by $K$. Let $\Gamma=\operatorname{Cay}(G, S), \Sigma=\operatorname{Cay}(G / N, N S / N)$, and $\Delta=\operatorname{Cos}(G, K, S)$, where $S$ is a generating set of the group $G$. Then, any element $g$ of $G$ can be uniquely expressed as $g=k n$ with $n \in N, k \in K$. Define the corresponding $\phi: k n \rightarrow(K n, N k)$ of $V(\Gamma)=G$ to $V(\Delta \times \Sigma)$. Then, it is easily verified that $\phi$ is a bijection, and we have the following result.

Theorem 2. The mapping $\phi$ is a homomorphism of the digraph $\Gamma$ to the digraph $\Delta \times \Sigma$.

\section{Proof. Omitted.}

As applications of Theorem 2, we consider the following two examples.

Example 1. Relating the butterfly network $B F_{q}$ to the de Bruijn network $D B_{2}^{q}$. Let $N=Z_{2}^{q}$ and $K=Z_{q}$. Then, $G=Z_{2} w r Z_{q}$ is a semidirect product of $N$ by $K$. Assuming $S=\left\{\left(0^{(q)}, 1\right),\left(0^{(q-1)} 1,1\right)\right\}$, we have $\Gamma=\operatorname{Cay}(G, S)=B F_{q}$, $\Sigma=\operatorname{Cay}(G / N, N S / N)=C_{q}$, where $C_{q}$ is a directed cycle of order $q$, and $\Delta=$ $\operatorname{Cos}(G, K, S)=D B_{2}^{q}$. Thus, we have a homomorphism $\phi$ from $B F_{q}$ to $D B_{2}^{q} \times C_{q}$. In fact, it is easily shown that it is an isomorphism and $B F_{q}=D B_{2}^{q} \times C_{q}$. 
Example 2. Relating the cube-connected cycles $C C C_{q}$ and the shuffleexchange network $S E_{2}^{q}$. Assume $N=Z_{2}^{q}, K=Z_{q}$, and $G=Z_{2} w r Z_{q}$, as in Example 1. Let $S^{\prime}=\left\{\left(0^{(q)}, 1\right),\left(0^{(q-1)} 1,0\right)\right\}$. Then, $\Gamma=\operatorname{Cay}\left(G, S^{\prime}\right)=C C C_{q}$, $\Sigma=\operatorname{Cay}\left(G / N, N S^{\prime} / N\right)=C_{q}^{+}$, where $C_{q}^{+}$is a directed cycle of order $q$ with a loop at every vertex, and $\Delta=\operatorname{Cos}\left(G, K, S^{\prime}\right)=S E_{2}^{q}$. In this way, we obtain a homomorphism of $C C C_{q}$ to $S E_{2}^{q} \times C_{q}^{+}$.

We now consider the broadcasting problem for interconnection networks. Broadcasting, a communication operation whereby a message is sent from one processor to all others, is a basic building block in the synthesis of parallel algorithms. The time to send a message from a processor to a neighboring one depends on the communication model assumed, with linear- and constant-time models being the two main choices. We assume the constant-time model, wherein communication between adjacent processors needs one time unit. Besides communication delay, other assumptions relating to the communication mode are needed. We assume that messages are sent in store-and-forward mode, where a processor cannot use the contents of a message, or send it on to another processor, until it has been received in its entirety. Given a connected graph $G$ (representing an interconnection network) and a message originator $u$, the broadcast time of the vertex $u, b_{M}(u)$, is the minimum time required to complete broadcasting from vertex $u$ under the model $M$. The broadcast time of $G$ under $M, b_{M}(G)$, is defined as the maximum broadcast time of any vertex $u$ in $G$; i.e., $b_{M}(G)=\max \left\{b_{M}(u) \mid u \in V(G)\right\}$. For more details, we refer the reader to [4]. Now let $G$ be a finite group and $K \leq G$. Assume that $\Gamma=\operatorname{Cay}(G, S)$ and $\Sigma=\operatorname{Cos}(G, K, S)$ for some generating set $S$ of $G$. For a communication model $M$, let $b_{M}\left(\Gamma_{K}\right)$ be the minimum time required to complete broadcasting in the vertices of $K$ from the identity element 1 (which is the message originator). One of our main results is as follows.

Theorem 3. $b_{M}(\Gamma) \leq b_{M}(\Delta)+b_{M}\left(\Gamma_{K}\right)$.

Proof. Omitted.

As applications of Theorem 3, we revisit Examples 1 and 2.

Example $1^{\prime}$. Consider the butterfly network $B F_{q}$ and the de Bruijn network $D B_{2}^{q}$. By Example 1, we know that $\Gamma=B F_{q}, \Delta=D B_{2}^{q}$. It is easily shown that $b_{M}\left(\Gamma_{K}\right) \leq b_{M}\left(C_{q}\right)$. Hence, we obtain $b_{M}(\Gamma) \leq b_{M}(\Delta)+b_{M}\left(C_{q}\right)$. In general, $b_{M}\left(C_{q}\right)$ is easily derived. For example, under the unit-time store-and-forward communication model, we have $b_{M}\left(C_{q}\right)=q-1$. So, we can obtain an upper bound on $b_{M}(\Gamma)$ when we know some upper bound on $b_{M}(\Delta)$. Similarly, any known lower bound on $b_{M}(\Gamma)$ leads to a corresponding lower bound for $b_{M}(\Delta)$.

Example $2^{\prime}$. Consider the cube-connected cycles $C C C_{q}$ and the shuffleexchange network $S E_{2}^{q}$. By Example 2, we have $b_{M}(\Gamma) \leq b_{M}(\Delta)+b_{M}\left(C_{q}\right)$, where $\Gamma=C C C_{q}, \Delta=S E_{2}^{q}$. So, the observations made in Example 1' apply here as well. The methods can be extended to the other communication problems and to undirected graphs. 
We assume that $\Gamma$ is a finite digraph (possibly with loops) having the vertex set $V(\Gamma)=\{1,2, \ldots, n\}$ and the arc set $E(\Gamma)=R$. Let $G=\operatorname{Aut}(\Gamma)$ be the automorphism group and $A(\Gamma)$ the adjacency matrix of the digraph $\Gamma$. Suppose that $R_{i}, 1 \leq i \leq r$, are orbits of the group $G$ acting on $V(\Gamma) \times V(\Gamma)$ such that $(x, y)^{g}=\left(x^{g}, y^{g}\right)$ for $x, y \in V(\Gamma)$ and $g \in G$. Let $\Gamma_{i}$ be the digraph with the vertex set $V(\Gamma)$ and the arc set $R_{i}$ for $1 \leq i \leq r$. Then, we have $G=\cap_{i=1}^{r} \operatorname{Aut}\left(\Gamma_{i}\right)$. Let $\Phi$ denote the empty set. It is easily verified that: (1) $A(\Gamma)=\sum\left\{A\left(\Gamma_{i}\right) \mid R \cap R_{i} \neq \Phi, 1 \leq i \leq r\right\}$, (2) $R_{i} \cap R_{j}=\Phi$ for $i \neq j$, (3) $R=\cup\left\{R_{i} \mid R \cap R_{i} \neq \Phi, 1 \leq i \leq r\right\}$. We denote the above as $\Gamma=\oplus\left\{\Gamma_{i} \mid R \cap R_{i} \neq\right.$ $\Phi, 1 \leq i \leq r\}$. Let $\Gamma$ be a Cayley digraph $\operatorname{Cay}(H, S)$ for a finite group $H$ and its generating set $S$. Then $H$ may be regarded as the left regular automorphism group of $\Gamma$. Because $H \leq G \leq \operatorname{Aut}\left(\Gamma_{i}\right)$, the digraph $\Gamma_{i}$ is a Cayley digraph per [4]. In fact, it is easily proven that $\Gamma_{i}=\operatorname{Cay}\left(H, S_{i}\right)$ and $S_{i}=\left\{\left(x^{g}\right)^{-1} y^{g} \mid(x, y) \in\right.$ $\left.R_{i}, g \in G\right\}$. Thus, we obtain the following factorization theorem and associated examples.

Theorem 4. $\operatorname{Cay}(H, S)=\oplus\left\{\operatorname{Cay}\left(H, S_{i}\right) \mid R \cap R_{i} \neq \Phi, 1 \leq i \leq r\right\}$.

Example 1" . For the butterfly network $B F_{q}$, we have: $B F_{q}=\operatorname{Cay}\left(Z_{2} w r Z_{q},\left\{\left(0^{(q)}, 1\right)\right\}\right) \oplus \operatorname{Cay}\left(Z_{2} w r Z_{q},\left\{\left(0^{(q-1)} 1,1\right)\right\}\right)$.

Example 2" . For the cube-connected cycles $C C C_{q}$, we have: $C C C_{q}=\operatorname{Cay}\left(Z_{2} w r Z_{q},\left\{\left(0^{(q)}, 1\right)\right\}\right) \oplus \operatorname{Cay}\left(Z_{2} w r Z_{q},\left\{\left(0^{(q-1)} 1,0\right)\right\}\right)$.

\section{Conclusion}

In this paper, we have supplied general theorems on homomorphism and broadcasting between Cayley digraphs and their coset graphs, and a factorization theorem on subgraphs of Cayley digraphs. We have also shown the applications of these results to some well-known interconnection networks: the butterfly network, the de Bruijn network, the cube-connected cycles network, and the shuffleexchange network. Many other useful directed and undirected networks can be similarly formulated and studied. Because of the generality of these theorems, we believe that they will have further applications to interconnection networks, providing an interesting area for further research. In particular, the design of scalable interconnection networks for parallel processing, offering the desirable properties of simple routing algorithms, balanced communication traffic, and resilience to node and link failures, can benefit from our results.

\section{References}

[1] Akers, S.B., Krishnamurthy, B.: A Group Theoretic Model for Symmetric Interconnection Networks. IEEE Trans. Computers, 38 (1989) 555-566

[2] Annexstein, F., Baumslag, M., Rosenberg, A.L.: Group Action Graphs and Parallel Architectures. SIAM J. Computing, 19 (1990) 544-569

[3] Biggs, N.: Algebraic Graph Theory. Cambridge University Press (1993) 
[4] Fraigniaud, P., Lazard, E.: Methods and Problems of Communication in Usual Networks. Discrete Applied Mathematics, 53 (1994) 79-133

[5] Heydemann, M.: Cayley Graphs and Interconnection Networks. In: Graph Symmetry: Algebraic Methods and Applications. (1997) 167-224

[6] Leighton, F.T.: Introduction to Parallel Algorithms and Architectures: Arrays, Trees, Hypercubes. Morgan Kaufmann (1992)

[7] Parhami, B.: Introduction to Parallel Processing: Algorithms and Architectures. Plenum (1999) 